\title{
Some Open Problems in Asymptotic Geometric Analysis
}

\author{
Bo'az Klartag and Elisabeth Werner \\ Communicated by Christina Sormani
}

\begin{abstract}
We describe four related open problems in asymptotic geometric analysis: the hyperplane conjecture, the isotropic constant conjecture, Sylvester's problem, and the simplex conjecture.
\end{abstract}

High-dimensional systems are frequent in mathematics and applied sciences, and understanding high-dimensional phenomena has become increasingly important. Asymptotic geometric analysis emerged as a new area that deals with exactly such phenomena. It is at the crossroads of such disciplines as functional analysis, convex geometry, and probability theory and bears connection to mathematical physics and theoretical computer science as well. The last two decades have seen tremendous growth in this area.

A major impulse for the theory is the hyperplane conjecture or slicing problem. Motivated by questions arising in harmonic analysis, it was first formulated by J. Bourgain and made known through the work of many people, such as K. Ball and V. Milman and A. Pajor. It asks if every centered convex body of volume 1 has a hyperplane section through the origin whose volume is greater than an absolute constant $c>0$ :

Hyperplane Conjecture. Every centered convex body $K$ of volume $|K|=1$ has a hyperplane section through the origin with volume greater than an absolute constant $c>0$, independent of dimension.

Bo'az Klartag is professor of mathematics at the Weizmann Institute and Tel Aviv University. His email address is k1 artagb@post .tau.ac.i1.

Elisabeth Werner is professor of mathematics at Case Western Reserve University. Her email address is eli sabeth. werner@case . edu.

For permission to reprint this article, please contact:

reprint-permission@ams.org.

DOI: http://dx.doi.org/10.1090/noti1685
Let us look at some easy examples for which the hyperplane conjecture holds. The first example is the Euclidean unit ball $B_{2}^{n}$, normalized to have volume 1:

$$
K=\frac{B_{2}^{n}}{\left|B_{2}^{n}\right|^{\frac{1}{n}}} .
$$

Every hyperplane section through the origin has $(n-1)$-dimensional volume

$$
\frac{\left|B_{2}^{n-1}\right|}{\left|B_{2}^{n}\right|^{\frac{n-1}{n}}}=\frac{\Gamma\left(1+\frac{n}{2}\right)^{\frac{n-1}{n}}}{\Gamma\left(1+\frac{n-1}{2}\right)},
$$

which, as $n$ approaches infinity, approaches $\sqrt{e}$.

The second example is the cube of volume 1 centered at the origin. Now every coordinate hyperplane slice has hypervolume 1 , a bit worse than the ball. The cube of course is the unit ball for the $l^{\infty}$ norm, just as the Euclidean ball was the unit ball for the $l^{2}$ norm.

More generally, for $1 \leq p<\infty$ consider the normalized unit ball in the $l^{p}$ norm:

$$
\|x\|_{p}=\left(\sum_{i=1}^{n}\left|x_{i}\right|^{p}\right)^{\frac{1}{p}} .
$$

Then every coordinate hyperplane slice has $(n-1)$ dimensional volume

$$
\frac{\left|B_{p}^{n-1}\right|}{\left|B_{p}^{n}\right|^{\frac{n-1}{n}}}=\frac{\Gamma\left(1+\frac{n}{p}\right)^{\frac{n-1}{n}}}{\Gamma\left(1+\frac{n-1}{p}\right)},
$$

which approaches $e^{1 / p}$. For the normalized $l_{p}^{n}$-unit balls, the maximal and minimal volume hyperplane sections are known. For instance, in the case of the normalized cube it was shown by $\mathrm{K}$. Ball that $\sqrt{2}$ is the maximum, and by H. Hadwiger and D. Hensley that 1 is the minimum.

At the heart of the hyperplane conjecture lies the question of distribution of volume in a high-dimensional convex body. It is now understood that the convexity assumption forces most of the volume of a body to be 


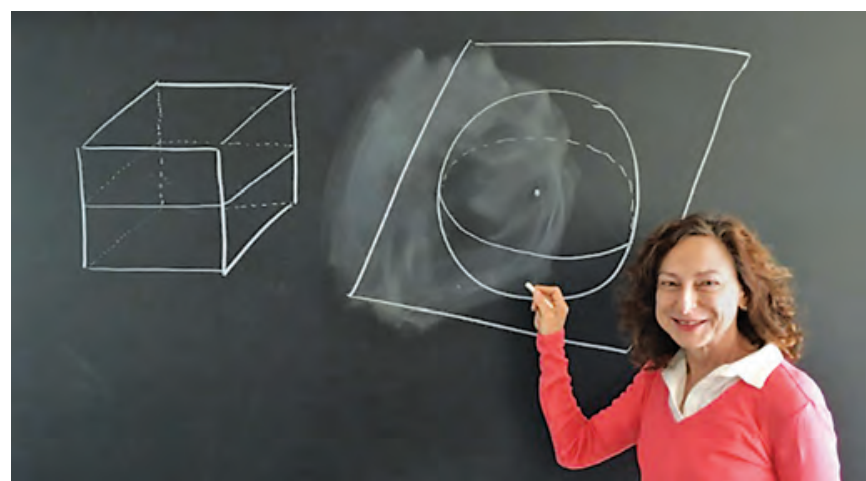

Elisabeth Werner shows hyperplane sections of the cube and the ball.

concentrated in some canonical way, and the main question is whether, under some natural normalization, the answer to many fundamental questions should be independent of the dimension. One such normalization, that in many cases facilitates the study of volume distribution, is the isotropic position, whose origin lies in classical mechanics of the nineteenth century. A convex body $K$ in $\mathbb{R}^{n}$ is called isotropic if it has volume 1 , is centered (has center of mass at the origin), and its inertia matrix is a multiple of the identity: there exists a constant $L_{K}>0$ such that for all $\theta$ in the Euclidean unit sphere $S^{n-1}$

$$
\int_{K}\langle x, \theta\rangle^{2} d x=L_{K}^{2} .
$$

It is always possible to put a convex body in isotropic position via an non-degenerate affine transformation, and the isotropic position is unique up to orthogonal transformations. For instance, the aforementioned normalized $l_{p}^{n}$-unit balls are in isotopic position. The relevance of isotropic position for the hyperplane conjecture comes from the fact that if $K$ is an isotropic convex body in $\mathbb{R}^{n}$, then all hyperplane sections through the origin have approximately the same volume and this volume is large enough if and only if $L_{K}$ is small enough: for every $\theta$ in the Euclidean unit sphere $S^{n-1}$ we have

$$
\frac{c_{1}}{L_{K}} \leq\left|K \cap \theta^{\perp}\right| \leq \frac{c_{2}}{L_{K}},
$$

where $c_{1}$ and $c_{2}$ are absolute constants and $\theta^{\perp}$ is the hyperplane section through the origin orthogonal to $\theta$. Of course, we can always put a convex body via an affine transformation in such a position that it has hyperplane sections that are arbitrarily large or arbitrarily small. The isotropic position prevents these very small or very large sections and puts investigation of the conjecture in the right setting. Thus, if we know a solution to the isotropic constant conjecture, which asks if there is an absolute constant $C$ such that for all dimensions and all convex bodies $K, L_{K} \leq C$, then, from (1) we know the solution to the hyperplane conjecture for isotropic bodies and the general case follows by a standard argument. In fact, the two conjectures are equivalent: assume that the hyperplane conjecture has an affirmative answer. If $K$ is isotropic, the above inequalities show that all sections $K \cap \theta^{\perp}$ have volume bounded by $c_{2} / L_{K}$ from above and since $\left|K \cap \theta^{\perp}\right| \geq c$ for at least one $\theta$, we get $L_{K} \leq c_{2} / c$.

It is known that $L_{K} \geq 1 / \sqrt{e}$ for all convex bodies $K$ in $\mathbb{R}^{n}$ and the minimum is attained for a Euclidean ball. There are recent connections to two classical problems. The first is that if among centrally-symmetric convex bodies $L_{K}$ is maximized for the cube then a classical Minkowski conjecture on lattices follows, as was shown in a series of works, the most recent one by A. Magazinov. The second is that if among all convex bodies $L_{K}$ is maximized for the simplex then an old conjecture by Mahler follows.

The best known upper bound to date for $L_{K}$ is due to Klartag, who showed that $L_{K} \leq C n^{1 / 4}$, removing the $\log$ factor in an earlier result by Bourgain that says $L_{K} \leq C n^{1 / 4} \log n$. For numerous special classes of convex bodies one can do better. For instance, one knows that $L_{B_{n}^{n}}$ is bounded above for all $1 \leq p \leq \infty$ by a constant independent of dimension. More generally, the isotropy constant is bounded for unconditional convex bodies. Those are convex bodies that are symmetric about the coordinate hyperplanes. And bounds are known for many more classes of convex bodies.

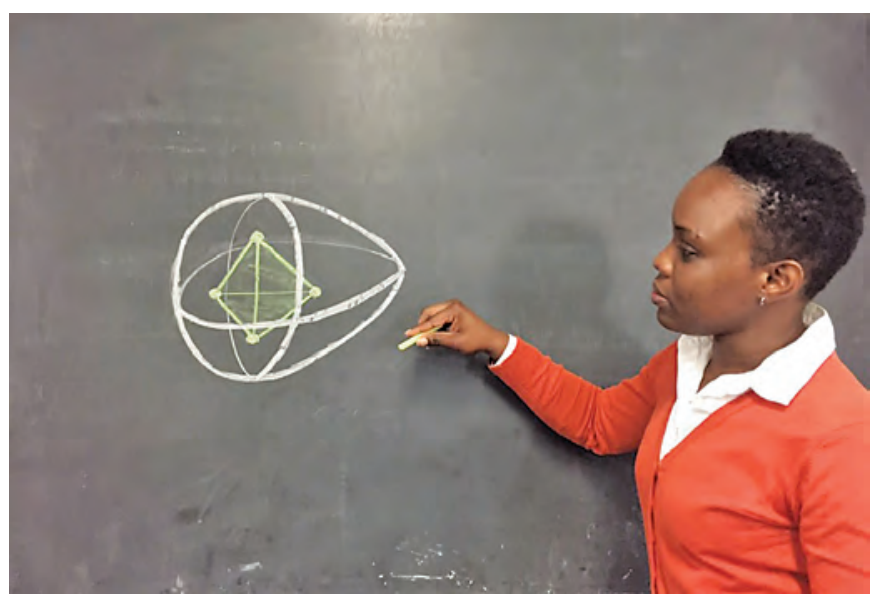

Nadiesha Burgess (Lehman College master's student) illustrates Sylvester's problem, which seeks extreme cases.

Several problems are related or equivalent to the hyperplane conjecture. We only mention Sylvester's problem and the simplex conjecture. The setup is as follows: One chooses random points $x_{1}, \ldots, x_{n+1}$ independently and uniformly distributed in an $n$-dimensional convex body $K$. Their convex hull conv $\left\{x_{1}, \ldots, x_{n+1}\right\}$ is a random simplex contained in $K$.

For every $p>0$, we consider

$m_{p}(K)$

$=\left(\frac{1}{|K|^{n+p+1}} \int_{K} \ldots \int_{K}\left|\operatorname{conv}\left\{x_{1}, \ldots, x_{n+1}\right\}\right|^{p} d x_{n+1} \ldots d x_{1}\right)^{1 / p}$.

Then $m_{p}(K)$ is invariant under nondegenerate affine transformations. Sylvester's problem asks to describe the affine classes of convex bodies for which $m_{p}(K)$ is minimized or maximized. It is known that for every 
$p>0, m_{p}(K) \geq m_{p}\left(B_{2}^{n}\right)$ with equality if and only if $K$ is an ellipsoid. In the opposite direction the problem is open if $n \geq 3$. The quantity $m_{1}(K)$ is the expectation of the normalized volume of a random simplex in $K$. The simplex conjecture states that for every convex body $K$ in $\mathbb{R}^{n}, m_{1}(K) \leq m_{1}\left(S_{n}\right)$, where $S_{n}$ is a simplex in $\mathbb{R}^{n}$. This conjecture has been verified only in the case $n=2$. If the simplex conjecture is correct, then the isotropic constant $L_{K} \leq C$ for every convex body $K$. So the simplex conjecture implies the hyperplane and isotropic constant conjectures.

Recent developments have led to investigations of properties of high-dimensional measures where the notion of independence has been replaced by geometric properties, such as convexity.

A good source for in-depth information, related problems, proofs, and references is the 2014 AMS Mathematical Surveys and Monographs Geometry of Isotropic of Isotropic Convex Bodies by S. Brazitikos, A. Giannopoulos, P. Valettas, and B. H. Vritsiou.

\section{Photo Credits}

Article photos courtesy of Elisabeth Werner.
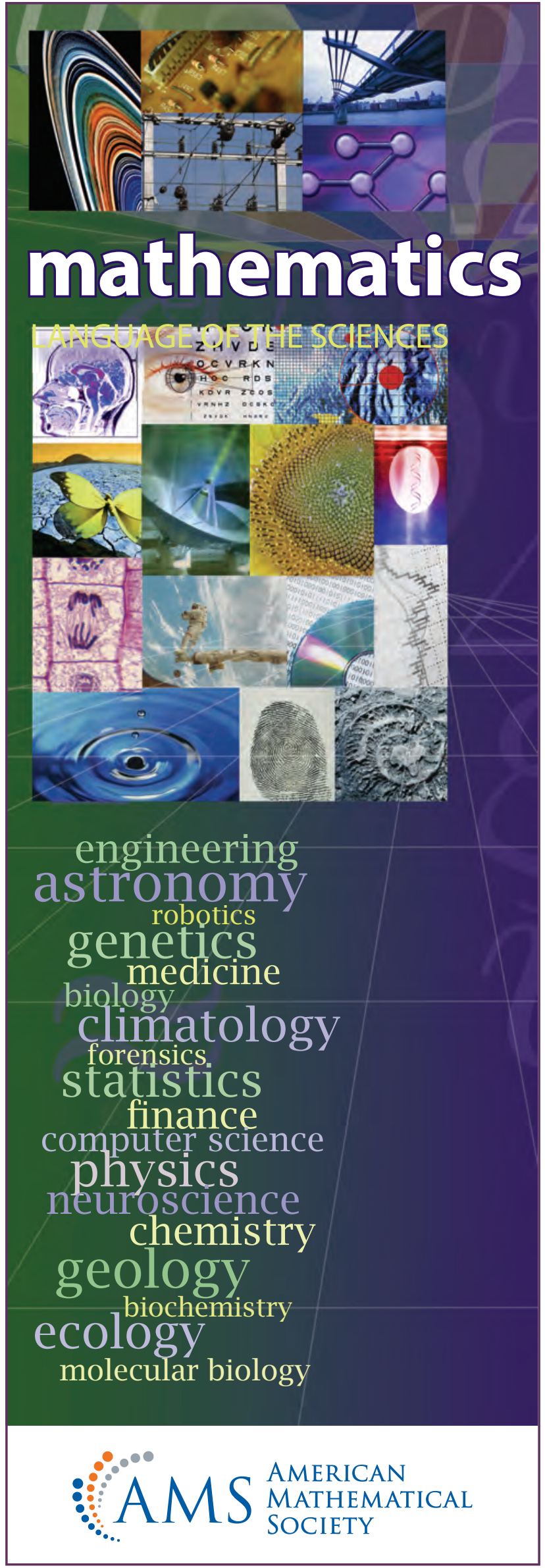